\title{
1 Contact time- and pH- dependent adhesion and cohesion of low molecular weight chitosan coated surfaces
}

$4 \quad$ Chanoong Lim ${ }^{\mathrm{a}, \dagger}$, Dong Woog Lee ${ }^{\mathrm{b}, \dagger}$, Jacob N. Israelachvili, YongSeok Jho ${ }^{\mathrm{c}, \mathrm{d}}$, and

6 aschool of Interdisciplinary Bioscience and Bioengineering, Pohang University of

7 Science and Technology (POSTECH), Pohang 790-784, South Korea

$8{ }^{b}$ Department of Chemical Engineering, University of California at Santa Barbara, CA 9 93106, USA.

10 CAsia Pacific Center for Theoretical Physics, and dDepartment of Physics, Pohang

11 University of Science and Technology, Pohang, Gyeongbuk, 790-784 South Korea

$12{ }^{\mathrm{e} S}$ School of Environmental Science and Engineering, ${ }^{\mathrm{f}}$ Integrative Biosciences and

13 Biotechnology, Pohang University of Science and Technology (POSTECH), Pohang

14 790-784, South Korea

15 * To whom correspondence should be addressed.

16 Email: dshwang@postech.ac.kr, Phone: +82(54) 279-9505, Fax: +82(54) 279-9519.

$17{ }^{\dagger}$ These authors equally contributed on this work 


\section{Abstract}

19 Low molecular weight chitosan (LMW chitosan, $\sim 5 \mathrm{kDa}$ ) potentially has many 20 desirable biomedical applications such as anti-microbial, anti-tumor, and anti21 diabetes. Unlike high molecular weight chitosan, LMW chitosan is easily dissolvable 22 in aqueous solutions even at neutral and basic $\mathrm{pH}$, but its dissolution mechanism is 23 not well understood. Here, we measured adhesion and cohesion of molecularly thin 24 LMW chitosan films in aqueous solutions in different buffer pHs (from 3.0 to 8.5) 25 using an surface forces apparatus (SFA). Interestingly, significantly lower adhesion 26 force was measured for LMW chitosan films compared to the high molecular weight 27 chitosan $(\sim 150 \mathrm{kDa})$ films. Not only the strength of adhesion is lower, but also the 28 critical contact time where adhesion starts to increase with contact time is longer. 29 The results from both the SFA and atomic force microscopy (AFM) indicate that, in 30 physiological and basic conditions, the low cohesion of LMW chitosan due to the 31 stiffness of the chain which cause strong electrostatic correlation energy penalty 32 when they are aggregated. Here, we propose the reduction in cohesion for shorter 33 chitosan (LMW chitosan) as an explanation of its high solubility of LMW chitosan in 34 physiological pHs.

35 Keywords: biomolecule; low molecular weight chitosan; surface forces apparatus; adhesion; cohesion; solubility 


\section{Introduction}

39 Chitin and chitosan are random copolymers composed of D-glucosamine and $N$ 40 acetyl-D-glucosamine units connected by $\beta-1,4$ linkages. The mole ratio of $D$ 41 glucosamine and $\mathrm{N}$-acetyl-D-glucosamine in the copolymers determines the two 42 terms, chitin and chitosan. Conventionally, the copolymer with higher mole fraction of 43 D-glucosamine unit is defined as chitosan, and the opposite case is called chitin. 44 Mole fraction of D-glucosamine unit in chitin and chitosan is defined as degree of 45 acetylation (DA) on D-glucosamine units. Generally, chitosan is obtained by 46 deacetylation and hydrolysis of chitin, which is the second most abundant 47 biomolecules in the nature (Chang, Tsai, Lee \& Fu, 1997; Ifuku et al., 2009; Mima, 48 Miya, Iwamoto \& Yoshikawa, 1983). Wide ranges of molecular weight of chitosan 49 (from $1 \mathrm{kDa}$ to $200 \mathrm{kDa}$ ) are produced from chitin, and their physical and biological 50 properties of chitosan differ as their molecular weight. To date, diverse forms of 51 chitosan have been used in many applications including healthcare, agriculture, 52 environmental engineering, medical engineering and cosmetics (Kumar, Muzzarelli, 53 Muzzarelli, Sashiwa \& Domb, 2004; Kurita, 1998, 2006; Rabea, Badawy, Stevens, 54 Smagghe \& Steurbaut, 2003; Ravi Kumar, 2000).

55 Unlike the chitosan, low molecular weight chitosan (LMW chitosan, $\leq 10 \mathrm{kDa}$ ) is 56 easily dissolvable even in buffer solutions at physiological pHs because of its shorter 57 chain length. The good solubility and low viscosity of LMW chitosan at physiological $58 \mathrm{pHs}$ have attracted public attention to utilize LMW chitosan in biomedical 59 applications. Indeed, a number of desirable biomedical applications of LMW chitosan 60 have been suggested, such as blood thinning, cholesterol-lowering, anti-oxidant, and 61 anti-microbial properties. (Busilacchi, Gigante, Mattioli-Belmonte, Manzotti \& 62 Muzzarelli, 2013; Felt, Buri \& Gurny, 1998; Hirano, Seino, Akiyama \& Nonaka, 1990; 63 Muzzarelli, 2009). However, many previous studies on biomedical applications of 64 LMW chitosan do not cover molecular mechanisms in detail (Muzzarelli, 2009).

65 Therefore, investigating molecular interactions and solubility of LMW chitosan in 66 aqueous buffers may help to unravel its mechanisms for the biomedical applications 67 eventually leading to a better and broader use of LMW chitosan. 
68 Surface forces apparatus (SFA) is one of the ideal biomechanical tools for measure

69 molecular interactions of bioactive molecules in wet conditions (Israelachvili, 2011)

70 with nano-Newton force sensitivity and angstrom distance resolution. It has been

71 used not only to measure normal adhesion forces (Lee, Banquy, Kristiansen,

72 Kaufman, Boggs \& Israelachvili, 2014), but also lateral friction forces (Lee, Banquy \&

73 Israelachvili, 2013). Recently, $\mathrm{pH}$ - and contact time- dependent interactions of

74 chitosan $(\mathrm{Mw} \sim 150 \mathrm{kDa}$ ) were measured with an SFA (Lee, Lim, Israelachvili \&

75 Hwang, 2013). Strong cohesion $\left(W_{\mathrm{co}} \sim 8.5 \mathrm{~mJ} / \mathrm{m}^{2}\right)$ between the two opposing

76 chitosan films was measured in acidic $\mathrm{pH}$, which is more than a half of the strongest,

77 previous reported, mussel wet adhesion (Hwang, Zeng, Lu, Israelachvili \& Waite,

78 2012; Hwang, Zeng, Masic, Harrington, Israelachvili \& Waite, 2010). However, the

79 interaction of LMW chitosan in aqueous solutions, and its relationship with higher

80 solubility of LMW chitosan compared to that of high molecular weight chitosan have

81 not been studied yet even though many desirable biomedical activities and potential

82 for biomedical applications of LMW chitosan.

83 In this study, we measured cohesion and adhesion of molecularly thin LMW 84 chitosan ( $M w \sim 5 \mathrm{kDa}$ ) films using an SFA in buffers with different pHs (from 3.0 to 85 8.5, Fig. 1). This work not only provides fundamental understandings on the 86 molecular mechanisms of LMW chitosan interactions in aqueous solutions at 87 different buffer pHs. 


\section{Materials and methods}

90

91

92

93

94

\subsection{Sample preparation and characterization}

Chitosan (LMW chitosan, Mw 5KDa) was purchased from Chitolife (Seoul, South Korea). The degree of acetylation (DA) of LMW chitosan ( 11\%) was determined by previously reported ${ }^{1} \mathrm{H}$ NMR base assay (de Alvarenga, Pereira de Oliveira \& Roberto Bellato, 2010; Hirai, Odani \& Nakajima, 1991). The polydispersity index of LMW chitosan measured by Dynamic light scattering was 0.3. LMW chitosan solution was prepared by dissolving LMW chitosan in $150 \mathrm{mM}$ acetic acid buffer ( $\mathrm{pH}$ 3.0), with final concentration of $50 \mu \mathrm{g} / \mathrm{mL}$. Prepared LMW chitosan solution was used throughout the experiments for surface forces measurements and chemical analysis. Three buffers with different pHs were prepared: $150 \mathrm{mM}$ acetic acid (pH 3.0), 150 $\mathrm{mM}$ sodium acetate buffer ( $\mathrm{pH}$ 6.5), and $150 \mathrm{mM}$ phosphate buffer $(\mathrm{pH}$ 8.5). All aqueous solutions were prepared in Milli-Q water (Millipore, Billeriaca, MA, USA) and filtered through $0.22 \mu \mathrm{m}$ PTFE filters (Millipore, Billeriaca, MA, USA).

\subsection{Surface preparation and LMW chitosan film deposition}

Muscovite mica surfaces (Grade \#1, S\&J Trading, Floral Park, NY, USA) were freshly cleaved under a dust free laminar flow hood. $55 \mathrm{~nm}$ of silver layer was deposited using a Joule effect vapor deposition. Thin and back-silvered mica sheets was glued (silver side down) on cylindrical glass disks using an epoxy glue (EPON $1004 \mathrm{~F} \circledast$, Exxon Chemicals, Irving, TX, USA). LMW chitosan was deposited on the back silvered mica surfaces according to previous reported procedures (Lee, Lim, Israelachvili \& Hwang, 2013). Briefly, $1 \mathrm{ml}$ of the prepared chitosan solution (50 $\mu \mathrm{g} / \mathrm{ml}$ in $150 \mathrm{mM}$ acetic acid, $\mathrm{pH} 3.0$ ) is dropped onto the mica surface and incubated for $20 \mathrm{~min}$ in a water saturated chamber. Then the surface was rinsed thoroughly with the acidic buffer solution (150 mM acetic acid, $\mathrm{pH} 3.0$ ) in order to remove unbound and weakly bound chitosan. 
118 The adhesion and cohesion forces of LMW chitosan films were determined using a 119 surface forces apparatus 2000 (Surforce LLC, Santa Barbara, CA, USA) in a 120 configuration that was previously reported in literature (Lee, Lim, Israelachvili \& 121 Hwang, 2013). Depending on the experiments, either two opposing LMW chitosan 122 coated surfaces (symmetric) or one LMW chitosan coated surface and one mica 123 surface (asymmetric) were transferred into the SFA chamber in cross-cylinder 124 geometry which corresponds to a sphere of radius $R(\sim 2 \mathrm{~cm})$ approaching a flat 125 surface. A droplet (50 $\mu$ l) of buffer $(\mathrm{pH} 3.0)$ was injected between the transferred 126 surfaces. The SFA chamber was sealed and saturated with water vapor during the 127 experiments. After mounting of the surfaces and injection of the buffer, the system 128 was allowed to equilibrate for $1 \mathrm{hr}$. All experiments were conducted at room 129 temperature $\left(T=23^{\circ} \mathrm{C}\right)$.

130 The interaction forces $F$ between the two opposing chitosan films in aqueous buffer 131 were measured as a function of the absolute surface separation distance $D$ between 132 two surfaces. $F$ was measured by the deflection of the double cantilever spring $(k=$ $1333030 \mathrm{~N} / \mathrm{m}$ ) of the lower surface and $D$ was determined using multiple beam 134 interferometry (MBI) (Israelachvili, 1973; Israelachvili et al., 2010; Israelachvili \& 135 Tabor, 1972). During a typical SFA force measurements, the two opposing surfaces 136 was brought toward each other by a motor driven micrometer to reach a "steric wall 137 distance" $\left(D_{\mathrm{sw}}\right)$ and kept in contact for a desired time, followed by separation. In the 138 repulsive regime of the force curve $(F / R>0)$, the interaction force $F$ can be converted 139 to the interaction energy $W$ by using Derjaguin approximation $(W=F / 2 \pi R)$ 140 (Israelachvili, 2011; Johnson, Kendall \& Roberts, 1971). Adhesion force $F_{\text {ad }}$ (for 141 asymmetric mode) and cohesion force $F_{\mathrm{co}}$ (for symmetric mode) are measured 142 during separation and the surfaces jump apart from adhesive contact when the 143 tensile load exceeds $F_{\mathrm{ad}}$ or $F_{\mathrm{co}}\left(F_{\mathrm{ad}} / R\right.$ and $F_{\mathrm{co}} / R$ defined as $-\min [F / R]$ to achieve 144 positive values). The adhesion energy per unit area $W_{\mathrm{ad}}$ can be calculated by 145 Johnson-Kendal-Roberts theory (Israelachvili, 2011; Johnson, Kendall \& Roberts, 146 1971), where $W_{\mathrm{ad}}=2 F_{\mathrm{ad}} / 3 \pi R$ for soft deformable surfaces. 
147 The surfaces were approached at a constant speed of $\sim 3 \mathrm{~nm} / \mathrm{s}$ to contact (up to $148 \mathrm{~F} / R \sim 4 \mathrm{mN} / \mathrm{m}$ ) and kept in contact for $5 \mathrm{sec}, 2 \mathrm{~min}, 10 \mathrm{~min}$, and $1 \mathrm{hr}$, followed by 149 separation to investigate the effect of contact time on cohesion/adhesion forces of 150 the LMW chitosan films. Force vs distance curves were measured at least 3 times at 151 each condition to confirm their reproducibility. In addition to the force measurement 152 under the $\mathrm{pH} 3.0$ buffer, the measurements were repeated after replacing the buffer 153 with an excess amount of $150 \mathrm{mM}$ sodium acetate buffer solution (pH 6.5) and 150 $154 \mathrm{mM}$ phosphate buffer solution $(\mathrm{pH} \mathrm{8.5)}$ to study the effects of $\mathrm{pH}$.

156 2.4. Surface topography study using Atomic force microscopy (AFM)

157 A LMW chitosan film was adsorbed onto freshly cleave mica surface by placing a 158 chitosan solution ( $10 \mu \mathrm{g} / \mathrm{ml}$ in $150 \mathrm{mM}$ acetic acid, $\mathrm{pH} 3.0)$ for $20 \mathrm{~min}$, and followed 159 by a thorough rinsing with the buffer solution (150 mM acetic acid, $\mathrm{pH}$ 3.0) in order to 160 remove unbound LMW chitosan. The LMW chitosan film on mica surface was 161 incubated for $1 \mathrm{hr}$ under three different $\mathrm{pHs}(3.0,6.5,8.5)$. The surfaces were 162 imaged using a Nanoscope III AFM (Veeco, Santa Barbara, CA, USA) using a silicon 163 nitride probe (Olympus, Tokyo, Japan), with a spring constant of $0.35 \mathrm{~N} \mathrm{~m}^{-1}$ in wet 164 conditions. Scannings were performed in tapping mode at room temperature ( 23ㄷ) 165 in three different $\mathrm{pHs}(3.0,6.5,8.5)$. 


\section{3. Results and discussion}

\section{3.1. LMW chitosan coating on mica surface}

169 The degree of acetylation (DA) determines physicochemical and biological properties 170 of Chitosan (LMW chitosan, Mw $\sim 5 \mathrm{kDa}$ ) (Cabrera, Boland, Cambier, Frettinger \& 171 Van Cutsem, 2010; Freier, Koh, Kazazian \& Shoichet, 2005). Solubility of LMW 172 chitosan in aqueous solutions, surface smoothness of the LMW chitosan film, and 173 cell proliferation on the LMW chitosan film decrease, when the DA of LMW chitosan 174 increases (Chatelet, Damour \& Domard, 2001). These phenomena suggest that the 175 DA of LMW chitosan is one of the key factors for interactions of LMW chitosan. 176 Therefore, prior to measuring interactions of LMW chitosan, the DA of LMW chitosan 177 was determined by ${ }^{1}$ NMR. The DA of LMW chitosan used in this study was $\sim 11 \%$, 178 which is slightly lower than that of previously studied chitosan (DA 19\%, Mw 150 $179 \mathrm{kDa}$ ) using an SFA (Lee, Lim, Israelachvili \& Hwang, 2013).

180 To monitor surface topographical changes with $\mathrm{pH}$, we prepared LMW chitosan 181 adsorbed mica surfaces (Fig. 2) following the procedure in materials and methods. 182 Atomic force microscopy (AFM) images of LMW chitosan coated mica in wet 183 conditions were generated after incubation with the buffer solutions at three different $184 \mathrm{pHs}(3.0,6.5$, and 8.5). AFM images revealed that a smooth chitosan film on mica 185 was roughened by $\mathrm{pH}$ elevation. The root-mean-square (RMS) roughness 186 determined by AFM for LMW chitosan coated surfaces at $\mathrm{pH} 3.0,6.5$, and 8.5 were $187 \sim 0.142 \mathrm{~nm}, \sim 0.204 \mathrm{~nm}$, and $\sim 0.236 \mathrm{~nm}$, respectively (Fig. 2).

\subsection{Interactions of LMW chitosan}

190 After LMW chitosan-adsorbed surface preparation, interaction forces between one 191 LMW chitosan film and bare mica (asymmetric mode, adhesion) or two opposing 192 LMW chitosan films (symmetric mode, cohesion) were measured using an SFA in the 193 aqueous buffer solutions at three different $\mathrm{pHs}$ (3.0, 6.5, and 8.5, Fig.1). Since 194 previous reported pKa value of LMW chitosan is about 6.5, the level of protonation in 
195 amine group of D-glucosamine units varies with $\mathrm{pH}$ : Glucosamine units in LMW 196 chitosan film on mica would be fully protonated at $\mathrm{pH} 3.0$, half of the glucosamine 197 units would be protonated at $\mathrm{pH} \mathrm{6.5}$, and the most of D-glucosamine units would be 198 deprotonated at $\mathrm{pH} 8.5$.

\subsubsection{Adhesion of LMW chitosan to mica (Asymmetric mode)}

201 For force measurement between the LMW chitosan film and negatively charged 202 bare mica, two surfaces were immersed in $150 \mathrm{mM}$ acetic acid buffer at pH 3.0 and 203 brought to together to reach a steric wall, $D_{\text {sw }}$. The $D_{\text {sw }}$ is defined as the two bare 204 mica surfaces separation distance $(D)$ when the distance $(D)$ does not apparently 205 change with the increase in the normal compressive load. Generally, the $D_{\text {sw }}$ 206 provides thickness of confined film trapped between two opposing mica surfaces in 207 the SFA experiment. Here, $D$ at $F / R=4 \mathrm{mN} / \mathrm{m}$ was designated as $D_{\mathrm{sw}}$. An adhesion 208 force $\left(F_{\mathrm{ad}} / R\right) \sim 12 \mathrm{mN} / \mathrm{m}$ and $D_{\mathrm{sw}} \sim 3 \mathrm{~nm}$ were measured after the contact time, $t_{\mathrm{ct}}$, of $2095 \mathrm{sec}$. When the $t_{\mathrm{ct}}$ was increased to $1 \mathrm{hr}$, the adhesion force monotonically 210 increased up to $F_{\mathrm{ad}} / R \sim 17 \mathrm{mN} / \mathrm{m}$ (Fig. $3 \mathrm{~A}$ and 5) and $D_{\mathrm{sw}}$ decreased down to $\sim 0.8$ $211 \mathrm{~nm}$ (Figure 3A) which is slightly thinner than that of chitosan ( 150 kDa) reported in 212 previous studies (Lee, Lim, Israelachvili \& Hwang, 2013). In addition, hysteresis was 213 observed between approach (which is purely repulsive) and separation force curves 214 (adhesive) even after very short contact time $\left(t_{\mathrm{ct}}<5 \mathrm{sec}\right)$. These results indicate that 215 a rearrangement and/or possible structural change - possibly from the relaxed two216 fold helix to the extended two-fold helix (Lertworasirikul, Tsue, Noguchi, Okuyama \& 217 Ogawa, 2003; Okuyama, Noguchi, Miyazawa, Yui \& Ogawa, 1997) - of LMW 218 chitosan molecules have occurred due to the external force in a confined space 219 which caused the LMW chitosan layer to be more compact and adhesive to the 220 opposing mica substrate. The adhesions of LMW chitosan to mica were $40 \%$ less 221 than those of previous studied higher molecular weight chitosan ( 150 kDa) to mica 222 (Lee, Lim, Israelachvili \& Hwang, 2013). Electrostatic interaction may account for the 223 initial adhesion force (when $t_{\mathrm{ct}}<t_{\mathrm{crit}}$ ) between positively charged chitosan film and 224 negatively charged mica. Poly-L-lysine, which has similar positive charged density 
225 and molecular weight ( $\mathrm{Mw} \sim 4 \mathrm{kDa}$ ) as LMW chitosan, showed similar initial attractive

226 force to mica surface $\left(F_{\mathrm{ad}} / R \sim 17 \mathrm{mN} / \mathrm{m}\right)$ in the previous SFA experiment (Afshar-Rad,

227 Bailey, Luckham, Macnaughtan \& Chapman, 1987). The increase in adhesion force 228 with contact time (when $t_{\mathrm{ct}}>t_{\text {crit }}$ ) is attributed to the hydrogen bonding between LMW 229 chitosan film and mica which is also related to the mobility of the hydrogen 230 donor/acceptor residues.

231 To examine effect of positive charges in chitosan, additional force measurements 232 between the LMW chitosan film and mica were performed at $\mathrm{pH} 6.5$ (around $\mathrm{pKa}$ of 233 LMW chitosan, Fig. 3B) and pH 8.5 (Fig. 3C). The adhesion of LMW chitosan film to 234 mica decreased monotonically with decreasing protonation of the amines in LMW 235 chitosan. Adhesion of LMW chitosan to mica at $\mathrm{pH} 6.5$ was reduced to approximately $23650 \%$ of its value at $\mathrm{pH} 3.0$, which is roughly corresponding to $50 \%$ deprotonation of 237 LMW chitosan. Moreover, the adhesion of LMW chitosan to mica at pH 8.5 was 238 significantly reduced. LMW chitosan and negatively charged mica could not interact 239 electrostatically anymore as most amine groups in LMW chitosan are deprotonated 240 at $\mathrm{pH}$ 8.5. A weak adhesion ( $\sim 3 \mathrm{mN} / \mathrm{m})$ with $1 \mathrm{hr}$ contact between the LMW chitosan 241 film and mica was measured and it is probably due to hydrogen bonding and/or van 242 der Waals forces between the LMW chitosan and mica. Taking the results above 243 together, it is clear that the strong adhesion at lower pHs arises from electrostatic 244 interaction between negatively charged mica and positively charged LMW chitosan 245 film. The LMW chitosan film thickness measured by $D_{\text {sw }}$ increased with an increase 246 in $\mathrm{pH}$ which was confirmed by both the SFA and the tapping mode atomic force 247 microscopy (AFM). The LMW chitosan films at higher $\mathrm{pH}$ bearing fewer positive 248 charges were less likely to bind to negatively charged mica surface and 249 consequently formed LMW chitosan aggregates on mica surfaces as observed in 250 AFM analysis (Fig. 2).

251 The persistent length of the polymer, $l_{t}$, which represents the flexibility of the chain, is 252 composed of two parts, the intrinsic part, $I_{\mathrm{p}}$, and the electrostatic part, $l_{\mathrm{e}}$, ie. $253 l_{\mathrm{t}} \simeq l_{\mathrm{p}}+l_{\mathrm{e}}$ (Skolnick \& Fixman, 1977). At low degree of polymerization (DP), the 254 intrinsic persistent length is linearly proportional to DP. For the LMW chitosan (DP 
$255 \sim 25$, and DA 11\%), the intrinsic persistent length is $l_{\mathrm{p}} \cong 6 \mathrm{~nm}$ (Rinaudo, 2006). In 256 this range, electrostatic persistent length is comparable with the intrinsic persistent 257 length, exhibiting about the value of $l_{\mathrm{e}} \cong 4.2 \mathrm{~nm}$. The total persistent length of LMW 258 chitosan $(\sim 10.2 \mathrm{~nm})$ is similar with its contour length $(\sim 15.6 \mathrm{~nm})$ at this $\mathrm{pH}$, and it 259 behaves as a rigid rod. On the highly anionic mica surface (surface charge density is 260 about $-2.0 \mathrm{e} / \mathrm{nm}^{2}$ ), if fully dissolved (Rojas, 2002), this multivalent rigid rod-like LMW 261 chitosan will be compactly condensed giving rise to charge inversion (Grosberg, 262 Nguyen \& Shklovskii, 2002). As seen from the experiment its thickness of the LMW 263 chitosan film is thinner than higher molecular weight chitosan film [20]. The 264 magnitude of the effective charge density can be approximated by Wigner-Seitz 265 crystal formation, $\frac{\sigma^{*}}{\sigma} \simeq \frac{\eta_{1}}{2 \pi a \sigma} \simeq 0.071$, where $\eta_{1}$ is the line charge density of rigid rod, $266 a$ is the cylindrical radius of rigid rod, $\sigma^{*}$ is the effect surface charge density of rigid $267 \mathrm{rod}$, and $\sigma$ is the surface charge density of rigid rod. At the end, two surfaces are 268 oppositely charged and the electrostatic interaction between them causes attraction. 269 The mechanism may differ for longer chain. In previous experiment, DP of high 270 molecular weight chitosan $(\sim 150 \mathrm{kDa})$ is about 750 (Lee, Lim, Israelachvili \& Hwang, 271 2013). In such case, the contour length of chain ( $343.2 \mathrm{~nm})$ is much longer than 272 persistence length, $l_{\mathrm{c}} \gg l_{\mathrm{t}}$. The radius of gyration is about $\sim 41 \mathrm{~nm}\left(R_{\mathrm{g}}=\right.$ $273 \sqrt{\frac{l_{\mathrm{c}} l_{\mathrm{t}}}{3}}$ ), which is much shorter than the contour length of high Mw chitosan, ie. we can 274 treat the high molecular weight chitosan as Gaussian chain. Thus, if we use the 275 same approach with short chain keeping the unit of persistence length as a single 276 rod carrying energy of $k_{\mathrm{B}} T$, it does not explain the difference in force between LMW 277 chitosan film and mica. Since the valence of charge within $l_{\mathrm{t}}$ is higher for longer 278 chain, more polymers are able to be condensed on the surface, although the 279 effective volume charge density of chain is reduced. This explains thicker $D_{s w}$ for 280 longer chain, but stronger attraction between film and mica. As increasing $\mathrm{pH}$, the 281 line charge density of the chain is decreased. Thus, the adhesion is strongest for $\mathrm{pH}$ 2823.0 , and weakest for $\mathrm{pH}$ 8.5. In case of $\mathrm{pH} 6.5$, perhaps the line charge density of 283 chitosan may not be enough to induce the charge inversion, but still the oppositely 284 charged polymers can mediate strong coupling attraction between two anionic mica 
surfaces.

\subsubsection{Cohesion of LMW chitosan (Symmetric mode)}

288

289

290

291

292

293

294

295

296

297

298

299

300

301

302

303

304

305

306

Figure 4 shows the force-distance profiles between LMW chitosan coated mica surfaces. The attractive force of the symmetric mode is significantly smaller than that of the asymmetric mode. Initially, when $t_{\mathrm{ct}}=5 \mathrm{sec}$, cohesion force between two opposing LMW chitosan was the largest at pH $6.5(\sim 1.8 \mathrm{mN} / \mathrm{m})$ and minimum at $\mathrm{pH}$ $3.0(\sim 0 \mathrm{mN} / \mathrm{m})$. As the $t_{\mathrm{ct}}$ was increased up to $1 \mathrm{hr}$, cohesion force increased for all pHs. Critical contact time $\left(t_{\text {crit }}\right)$, when cohesion force started to increase, was only observed at $\mathrm{pH}$ 3.0. Cohesion between the LMW chitosan films was much less than adhesion of the LMW chitosan to mica due to the electrostatic interaction. The attractive forces between the two LMW chitosan films could be largely categorized as van der Waals force, hydrophobic interactions, hydrogen bond and electrostatic force. Of these, hydrogen bonds between opposing LMW chitosan films are formed due to the presence of amine group, hydroxyl group, and acetyl group. Participating hydrogen bonds in the LMW chitosan cohesion varies on the buffer $\mathrm{pH}$. Critical contact time $\left(t_{\mathrm{ct}}\right)$ was required for $\mathrm{pH} 3.0$ as we observed in high molecular weight chitosan cohesion (Lee, Lim, Israelachvili \& Hwang, 2013), which is likely due to time required to overcome strong electrostatic repulsion between fully protonated amine groups and hydrogen bonding groups to meet each other between the opposing LMW chitosan films. In contrast to previously studied cohesion of high molecular weight chitosan $\left(W_{\text {co }} \sim 8.5 \mathrm{~mJ} / \mathrm{m}^{2}\right)$ at $\mathrm{pH} 3.0$, even after the $1 \mathrm{hr}$ contact time, cohesion of LMW chitosan at $\mathrm{pH} 3.0$ was significantly small $\left(W_{\text {co }} \sim 0.22 \mathrm{~mJ} / \mathrm{m}^{2}\right)$.

At $\mathrm{pH}$ 3.0, due to fully protonated amine groups, two mica surfaces are overcharged to induce charge inversion. From the AFM image (Figure 2), we learned that the LMW chitosan film coated on the mica surface is very smooth (RMS roughness $\sim 0.142 \mathrm{~nm}$ ), which excludes the possible LMW chitosan aggregate domain formation and their consequent correlated attraction (Jho, Brewster, Safran \& Pincus, 2011; Meyer, Lin, Hassenkam, Oroudjev \& Israelachvili, 2005; Perkin, Kampf \& Klein, 2006). As a result, two over-charged surfaces repel each other by the electrostatic 
315 interaction at long distance. Imposing external force to contact two coated surfaces,

316 the hydrogen bond, which is short ranged and attractive, can be established whereas

317 the electrostatic interaction is still repulsive. So, it takes time to connect enough

318 number of hydrogen bonds by changing its solution secondary structure from relaxed

319 two-fold helix to extend two-fold helix, in order to overcome the electrostatic

320 repulsion (Lertworasirikul, Tsue, Noguchi, Okuyama \& Ogawa, 2003; Okuyama,

321 Noguchi, Miyazawa, Yui \& Ogawa, 1997). This explains the existence of $t_{\text {crit }}$ at $\mathrm{pH} 3.0$

322 and when $t_{\mathrm{ct}}<t_{\text {crit, }}$, only a small fraction of the amine groups of LMW chitosan

323 molecules may participate in hydrogen bonds.

324 Interestingly, strongest adhesion is observed for $\mathrm{pH} 6.5$ (Figure 4B). At this $\mathrm{pH}$, 325 attraction is steadily increased starting from the short contact time, $t_{\mathrm{ct}}=5 \mathrm{sec}$, which 326 indicates $t_{\text {crit }}<5 \mathrm{sec}$. The AFM image of this buffer system presented irregular 327 surface because of partly aggregated LMW chitosan molecules. At pH 6.5, the 328 charge density of the LMW chitosan is reduced, and as well as the persistent length.

329 This implies that the LMW chitosan chain weakly interacts with mica surface and not 330 likely to form a compact structure on the mica surface as in $\mathrm{pH} 3.0$. Charge inversion 331 may be suppressed or reduced significantly, resulting in LMW chitosan aggregate 332 formation on mica. These positive charged LMW chitosan aggregates may be 333 correlated to mediate long range electrostatic attraction between two opposing LMW 334 chitosan coated surfaces (Sagui \& Darden, 1999; Stone, 2013), not requiring long 335 contact time for adhesion. Hydrogen bond is also easier to be formed while two 336 surfaces are in contact. Thus, about $\sim 0.4 \mathrm{~mJ} / \mathrm{m}^{2}$ appeared in initial contact time is 337 supposed to be mainly electrostatic bonding. This interaction can be comparable to $338 \sim 1.1 \mathrm{~mJ} / \mathrm{m}^{2}$ that of initial interaction at asymmetric case. After the LMW chitosan 339 films are in contact, the attraction gradually increases by the formation of new 340 hydrogen bond as the contact time increase.

341 At the $\mathrm{pH} 8.5$ (Figure 4C), LMW chitosan is mostly neutral due to the deprotonation 342 of most amine groups. It may be considered as van der Waals interaction which 343 contributes to the attraction before the $t_{\text {crit. }}$. After $t_{\text {crit }}$, hydrogen bonds start to be 344 formed. The cohesion increase after $t_{\text {crit }}$ is about $\sim 0.4 \mathrm{~mJ} / \mathrm{m}^{2}$ at $\mathrm{pH} 8.5$ which is 
345 bigger than the cohesion increase $\left(\sim 0.1 \mathrm{~mJ} / \mathrm{m}^{2}\right)$ at $\mathrm{pH}$ 6.5. It means that number of

346 molecular groups and/or molecules which participate in hydrogen bonding at the $\mathrm{pH}$

$347 \quad 6.5$ are less than $\mathrm{pH} 8.5$.

\subsection{Molecular weight effects upon the chitosan interaction and solubility}

350 The adhesion of LMW chitosan $(\sim 5 \mathrm{kDa})$ to mica, mediated by electrostatic 351 interaction, were $\sim 40 \%$ less than that of previous studied higher molecular weight 352 chitosan ( $\sim 150 \mathrm{kDa})$ to mica (Lee, Lim, Israelachvili \& Hwang, 2013) whereas the 353 cohesion of LMW chitosan coated films are reduced over 1/10 (Figure S1). These 354 may be attributed to the valence and flexibility of the higher molecular weight 355 chitosan. Molecular weight effects upon adhesion and cohesion have been studied 356 with various types of polymers (Choi, Zurawsky \& Ulman, 1999; Deruelle, Leger \& 357 Tirrell, 1995; Jenkins, Meredith \& Wilker, 2013). Generally, increase in molecular 358 weight induces additional chain entanglements (Choi, Zurawsky \& Ulman, 1999). In 359 chitosan case, participation of the hydrogen bond in chitosan may be enhanced, too. 360 However, a more likely explanation of our results is that the persistent length of the 361 chain is longer for the higher molecular weight chitosan, which mediates stronger 362 electrostatic attraction with less entropic penalty. For this reason, longer chain can 363 mediate not only stronger electrostatic interaction, but also induce more hydrogen 364 bonds due to its flexibility. In contrast, for short chain LMW chitosan, whose contour 365 length is shorter than persistence length, they will be very rigid, and be compactly 366 packed on the oppositely charged surface. As a consequence, the overcharged 367 LMW chitosan coated films in symmetric mode may repel each other, therefore it 368 reduces the cohesion significantly, while the overcharged LMW chitosan film in 369 asymmetric mode still shows an attraction although the magnitude is slightly smaller 370 than high molecular weight case. At higher $\mathrm{pH}$, the entropy of the chain increases 371 which loosen the attachment of chitosan to the mica surface, and eventually, it 372 reduces the electrostatic repulsion between chitosan molecules, and makes easier 373 them to be aggregated seen in AFM image. The result is consistent with long chain 374 case. At the end, they are easier to overcome the electrostatic repulsion, and to 
375 make hydrogen bonds as increasing $\mathrm{pH}$.

376 As stated before, main advantage of LMW chitosan over higher molecular weight 377 chitosan is good solubility in the physiological $\mathrm{pH}$ range which is directly implied by 378 our results. It has been known that the solubility has an inverse relation with 379 cohesion energy, for example the Hildebrand solubility parameter depends on 380 inverse square root of cohesive energy density (Barton, 1991; Hansen, 2012; Kamlet, 381 Carr, Taft \& Abraham, 1981). Thus, the reduction of cohesion measured in LMW 382 chitosan will lead higher solubility of LMW chitosan in physiological pH range. 


\section{4. Conclusion}

384 We have measured the adhesion and cohesion forces of low molecular weight 385 chitosan (LMW chitosan, $5 \mathrm{kDa}$ ) at different pHs and contact times, and compared 386 results with high molecular weight chitosan $(\sim 150 \mathrm{kDa})$ which has about 30 times 387 longer chain length. When fully protonated, LMW chitosan is very stiff rod-like 388 cationic chain. Two opposing LMW chitosan films are well separated due to the 389 strong electrostatic repulsion in long distance. It requires huge external force to 390 overcome the repulsion, which significantly reduces the cohesion of LMW chitosan. 391 After the LMW chitosan films are brought into contact, it requires a long time to 392 establish hydrogen bond network between two opposing surfaces. The weaker 393 cohesion compared to the higher molecular weight chitosan explains the good 394 solubility of LMW chitosan within physiological $\mathrm{pH}$ range. The contour length of the 395 high molecular weight chitosan is much longer than that of the persistence length 396 which results in the easier formation of the hydrogen bonds and higher possibility of 397 the chain entanglement. Thus, longer chain can have stronger cohesion with shorter 398 critical contact time, which reduces the solubility of high molecular weight chitosan. 399 Contact time- and $\mathrm{pH}$ - dependent interaction of LMW chitosan help to accelerate its 400 future application.

\section{Acknowledgements}

403 This work was supported by the Marine Biomaterials Research Center under Marine 404 Biotechnology Program, Ministry of Oceans and Fisheries Affairs, Korea, and US 405 Department of Energy, Office of Basic Energy Sciences, Division of Materials 406 Sciences under Award DE-FG02-87ER-45331 (J.N.I. for the instrument modification 407 of the Surface Forces Apparatus for the adhesion measurements and D.W.L. for the 408 adhesion measurements). We also acknowledge the National Research Foundation 
409 of Korea Grant funded by the Ministry of Science, ICT and Future Planning (MSIP)

410 (NRF-C1ABA001-2011-0029960 \& NRF-2014R1A2A2A01006724). 


\section{References}

413 Afshar-Rad, T., Bailey, A., Luckham, P., Macnaughtan, W., \& Chapman, D. (1987).

414 Forces between poly-L-lysine of molecular weight range $4,000-75,000$ adsorbed on 415 mica surfaces. Colloids and surfaces, 25(2), 263-277.

416 Barton, A. F. (1991). CRC handbook of solubility parameters and other cohesion 417 parameters. CRC press.

418 Busilacchi, A., Gigante, A., Mattioli-Belmonte, M., Manzotti, S., \& Muzzarelli, R. A. 419 (2013). Chitosan stabilizes platelet growth factors and modulates stem cell 420 differentiation toward tissue regeneration. Carbohydrate Polymers, 98(1), 665-676.

421 Cabrera, J.-C., Boland, A., Cambier, P., Frettinger, P., \& Van Cutsem, P. (2010). 422 Chitosan oligosaccharides modulate the supramolecular conformation and the 423 biological activity of oligogalacturonides in Arabidopsis. Glycobiology, 20(6), 775-786. 424 Chang, K. L. B., Tsai, G., Lee, J., \& Fu, W.-R. (1997). Heterogeneous N425 deacetylation of chitin in alkaline solution. Carbohydrate Research, 303(3), 327-332.

426 Chatelet, C., Damour, O., \& Domard, A. (2001). Influence of the degree of acetylation 427 on some biological properties of chitosan films. Biomaterials, 22(3), 261-268.

428 Choi, G. Y., Zurawsky, W., \& Ulman, A. (1999). Molecular weight effects in adhesion. 429 Langmuir, 15(24), 8447-8450.

430 de Alvarenga, E. S., Pereira de Oliveira, C., \& Roberto Bellato, C. (2010). An 431 approach to understanding the deacetylation degree of chitosan. Carbohydrate 432 Polymers, 80(4), 1155-1160.

433 Deruelle, M., Leger, L., \& Tirrell, M. (1995). Adhesion at the solid-elastomer interface: 434 influence of the interfacial chains. Macromolecules, 28(22), 7419-7428.

435 Felt, O., Buri, P., \& Gurny, R. (1998). Chitosan: A unique polysaccharide for drug 
delivery. Drug Development and Industrial Pharmacy, 24(11), 979-993.

437 Freier, T., Koh, H. S., Kazazian, K., \& Shoichet, M. S. (2005). Controlling cell 438 adhesion and degradation of chitosan films by $<\mathrm{i}>\mathrm{N}</ \mathrm{i}>$-acetylation. Biomaterials, $439 \quad 26(29), 5872-5878$.

440 Grosberg, A. Y., Nguyen, T., \& Shklovskii, B. (2002). Colloquium: the physics of 441 charge inversion in chemical and biological systems. Reviews of modern physics, $44274(2), 329$.

443 Hansen, C. M. (2012). Hansen solubility parameters: a user's handbook. CRC press.

444 Hirai, A., Odani, H., \& Nakajima, A. (1991). Determination of degree of deacetylation 445 of chitosan by $1 \mathrm{H}$ NMR spectroscopy. Polymer Bulletin, 26(1), 87-94.

446 Hirano, S., Seino, H., Akiyama, Y., \& Nonaka, I. (1990). Chitosan: a biocompatible 447 material for oral and intravenous administrations. Progress in biomedical polymers 448 (pp. 283-290): Springer.

449 Hwang, D. S., Zeng, H., Lu, Q., Israelachvili, J., \& Waite, J. H. (2012). Adhesion 450 mechanism in a DOPA-deficient foot protein from green mussels. Soft matter, 8(20), $451 \quad 5640-5648$

452 Hwang, D. S., Zeng, H., Masic, A., Harrington, M. J., Israelachvili, J. N., \& Waite, J. H. 453 (2010). Protein-and metal-dependent interactions of a prominent protein in mussel 454 adhesive plaques. Journal of biological chemistry, 285(33), 25850-25858.

455 Ifuku, S., Nogi, M., Abe, K., Yoshioka, M., Morimoto, M., Saimoto, H., \& Yano, H. 456 (2009). Preparation of chitin nanofibers with a uniform width as a-chitin from crab 457 shells. Biomacromolecules, 10(6), 1584-1588.

458 Israelachvili, J. (1973). Thin film studies using multiple-beam interferometry. Journal 
of Colloid and Interface Science, 44(2), 259-272. Israelachvili, J., Min, Y., Akbulut, M., Alig, A., Carver, G., Greene, W., Kristiansen, K., Meyer, E., Pesika, N., Rosenberg, K., \& Zeng, H. (2010). Recent advances in the surface forces apparatus (SFA) technique. Reports on Progress in Physics, 73(3), 16. Israelachvili, J., \& Tabor, D. (1972). The measurement of van der Waals dispersion 464 forces in the range 1.5 to $130 \mathrm{~nm}$. Proceedings of the Royal Society of London. A. 465 Mathematical and Physical Sciences, 331(1584), 19-38. Israelachvili, J. N. (2011). Intermolecular and surface forces: revised third edition. 467 Academic press. Jenkins, C. L., Meredith, H. J., \& Wilker, J. J. (2013). Molecular weight effects upon the adhesive bonding of a mussel mimetic polymer. ACS applied materials \& 470 interfaces, 5(11), 5091-5096.

471 Jho, Y., Brewster, R., Safran, S., \& Pincus, P. (2011). Long-range interaction between 472 heterogeneously charged membranes. Langmuir, 27(8), 4439-4446.

473 Johnson, K., Kendall, K., \& Roberts, A. (1971). Surface energy and the contact of 474 elastic solids. Proceedings of the Royal Society of London. A. Mathematical and 475 Physical Sciences, 324(1558), 301-313.

476 Kamlet, M. J., Carr, P. W., Taft, R., \& Abraham, M. H. (1981). Linear solvation energy 477 relationships. 13. Relationship between the Hildebrand solubility parameter,. delta. H, 478 and the solvatochromic parameter,. pi. *. Journal of the American Chemical Society, $479 \quad 103(20), 6062-6066$.

480 Kumar, M. R., Muzzarelli, R. A., Muzzarelli, C., Sashiwa, H., \& Domb, A. (2004). 481 Chitosan chemistry and pharmaceutical perspectives. Chemical reviews, 104(12), 
6017-6084.

483 Kurita, K. (1998). Chemistry and application of chitin and chitosan. Polymer 484 Degradation and Stability, 59(1-3), 117-120.

485 Kurita, K. (2006). Chitin and chitosan: Functional biopolymers from marine 486 crustaceans. Marine Biotechnology, 8(3), 203-226.

487 Lee, D. W., Banquy, X., \& Israelachvili, J. N. (2013). Stick-slip friction and wear of 488 articular joints. Proceedings of the National Academy of Sciences, 110(7), E567489 E574.

490 Lee, D. W., Banquy, X., Kristiansen, K., Kaufman, Y., Boggs, J. M., \& Israelachvili, J. 491 N. (2014). Lipid domains control myelin basic protein adsorption and membrane 492 interactions between model myelin lipid bilayers. Proceedings of the National 493 Academy of Sciences, 111(8), E768-E775.

494 Lee, D. W., Lim, C., Israelachvili, J. N., \& Hwang, D. S. (2013). Strong Adhesion and 495 Cohesion of Chitosan in Aqueous Solutions. Langmuir, 29(46), 14222-14229.

496 Lertworasirikul, A., Tsue, S.-i., Noguchi, K., Okuyama, K., \& Ogawa, K. (2003). Two 497 different molecular conformations found in chitosan type II salts. Carbohydrate 498 Research, 338(11), 1229-1233.

499 Meyer, E. E., Lin, Q., Hassenkam, T., Oroudjev, E., \& Israelachvili, J. N. (2005). 500 Origin of the long-range attraction between surfactant-coated surfaces. Proceedings 501 of the National Academy of Sciences of the United States of America, 102(19), 6839$502 \quad 6842$.

503 Mima, S., Miya, M., Iwamoto, R., \& Yoshikawa, S. (1983). Highly deacetylated 504 chitosan and its properties. Journal of Applied Polymer Science, 28(6), 1909-1917. 
505 Muzzarelli, R. A. (2009). Chitins and chitosans for the repair of wounded skin, nerve, 506 cartilage and bone. Carbohydrate Polymers, 76(2), 167-182.

507 Okuyama, K., Noguchi, K., Miyazawa, T., Yui, T., \& Ogawa, K. (1997). Molecular and 508 crystal structure of hydrated chitosan. Macromolecules, 30(19), 5849-5855.

509 Perkin, S., Kampf, N., \& Klein, J. (2006). Long-range attraction between charge510 mosaic surfaces across water. Physical review letters, 96(3), 038301.

511 Rabea, E. I., Badawy, M. E. T., Stevens, C. V., Smagghe, G., \& Steurbaut, W. (2003). 512 Chitosan as antimicrobial agent: Applications and mode of action. 513 Biomacromolecules, 4(6), 1457-1465.

514 Ravi Kumar, M. N. (2000). A review of chitin and chitosan applications. Reactive and 515 functional polymers, 46(1), 1-27.

516 Rinaudo, M. (2006). Chitin and chitosan: Properties and applications. Progress in 517 Polymer Science, 31(7), 603-632.

518 Rojas, O. J. (2002). Adsorption of polyelectrolytes on mica. Encyclopedia of Surface 519 and Colloid Science, 1, 517.

520 Sagui, C., \& Darden, T. A. (1999). Molecular dynamics simulations of biomolecules:

521 long-range electrostatic effects. Annual review of biophysics and biomolecular 522 structure, $28(1), 155-179$.

523 Skolnick, J., \& Fixman, M. (1977). Electrostatic persistence length of a wormlike 524 polyelectrolyte. Macromolecules, 10(5), 944-948.

525 Stone, A. (2013). The theory of intermolecular forces. Oxford University Press. 


\section{Figure legend}

530 Fig.1. A. Chemical structures of chitosan molecule. Chitosan is copolymers of D531 glucosamine $(m)$ and $N$-acetyl-D-glucosamine $(n)$ unit. $(m>n)$ B. Schematic figures of

532 separated mica surfaces with distance, $D$, in asymmetric mode (left), contacted mica 533 surfaces mode with a steric wall distance $\left(D_{\mathrm{sw}}\right)$ in asymmetric mode (Right). C. 534 Schematic figures of separated mica surfaces in symmetric mode. $R$ is the radius of 535 the surfaces.

Fig. 2. AFM tapping mode images of low molecular weight chitosan (LMW chitosan) 538 films deposited at pH3.0 on freshly cleaved muscovite mica and incubated for $1 \mathrm{hr}(\mathrm{A})$ 539 at $\mathrm{pH} 3.0,(\mathrm{~B})$ at $\mathrm{pH} 6.5$, and $(\mathrm{C})$ at $\mathrm{pH} 8.5$.

541 Fig. 3. Low molecular weight chitosan (LMW chitosan) adhesion to mica 542 (asymmetric mode). Force - distance curves between low molecular weight chitosan 543 and mica in $(\mathrm{A}) \mathrm{pH} 3.0,(\mathrm{~B}) \mathrm{pH} \mathrm{6.5}$, and $(\mathrm{C}) \mathrm{pH}$ 8.5. All profiles are the representative 544 results at each $\mathrm{pH}$ condition.

Fig. 4. Force - distance curves between two opposing low molecular weight chitosan 547 (LMW chitosan) films in (A) $\mathrm{pH} 3.0$, (B) $\mathrm{pH} \mathrm{6.5,} \mathrm{and} \mathrm{(C)} \mathrm{pH} 8.5$ (symmetric mode). All 548 profiles are the representative results at each $\mathrm{pH}$ condition.

550 Fig. 5. (A) Low molecular weight chitosan (LMW chitosan) adhesion to mica 551 depending on contact time and $\mathrm{pH}$, and (B) Cohesion of LMW chitosan depending 552 on contact time and $\mathrm{pH}$. 
A.

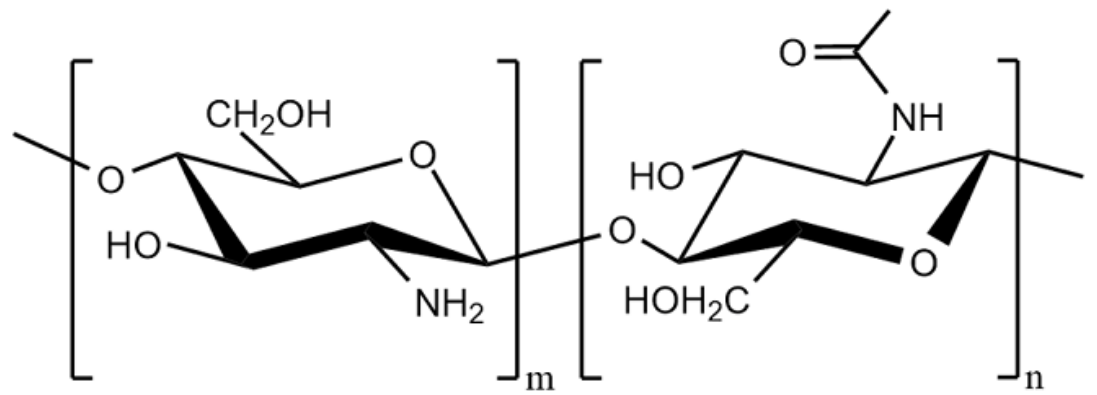

B.

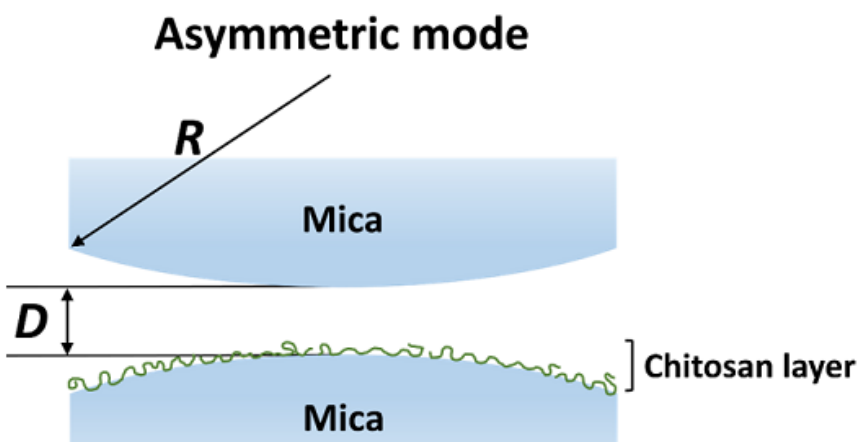

C.

Symmetric mode

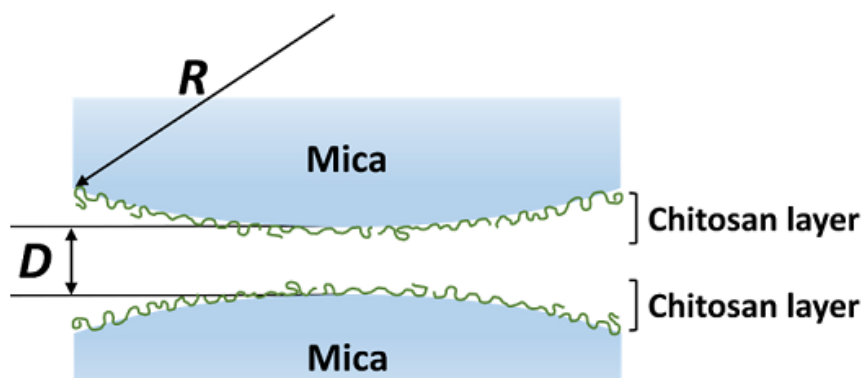

contact

Symmetric mode

In contact

contact
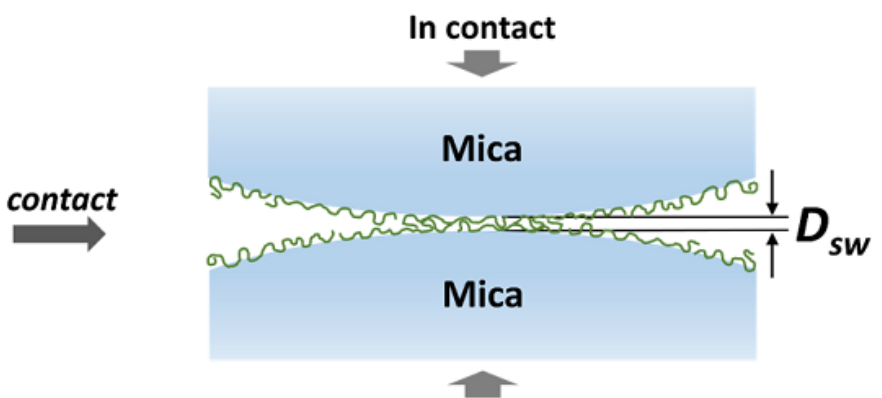

Asymmetric mode

In contact

Mica

Mica sw

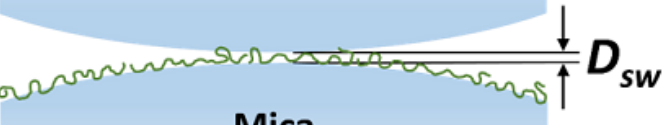




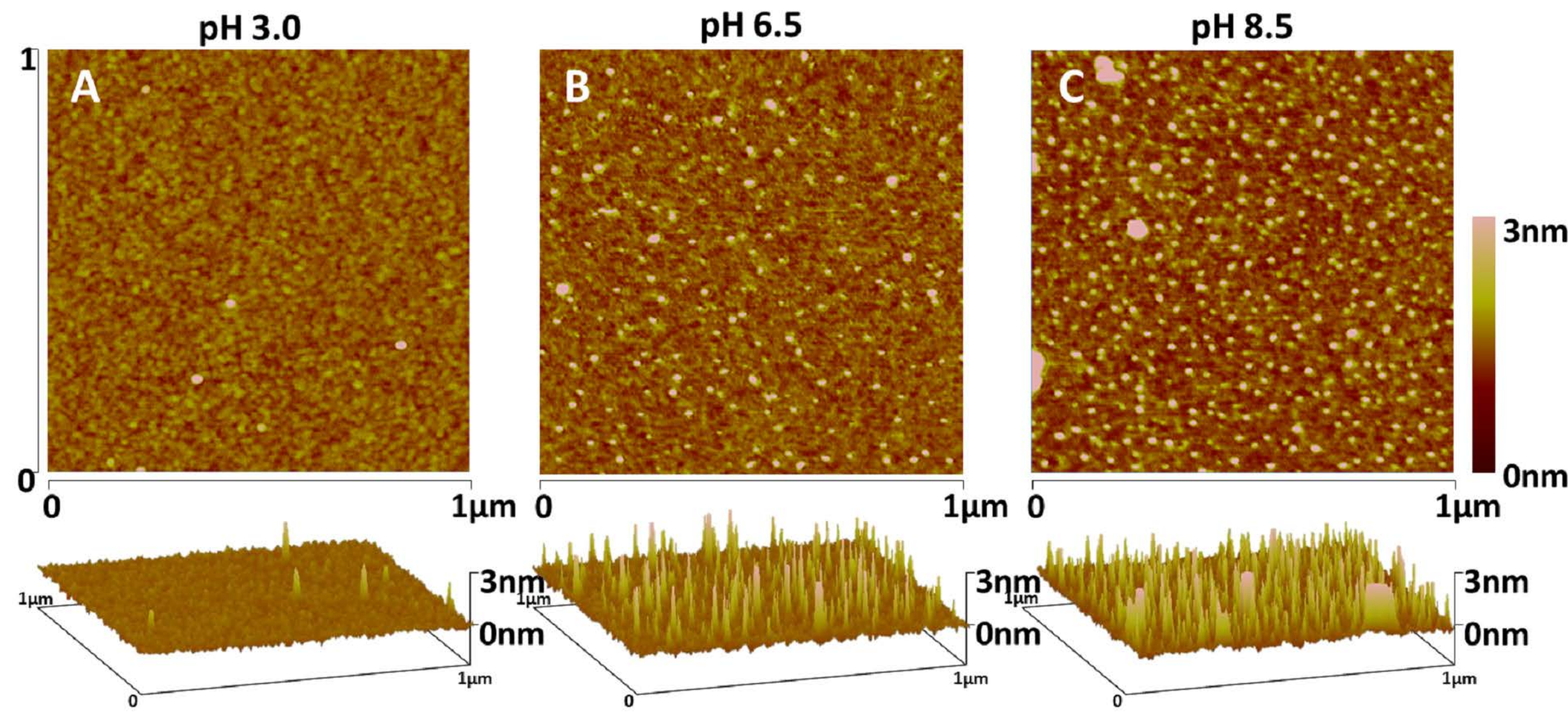



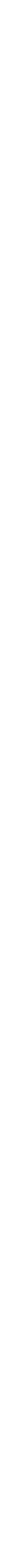


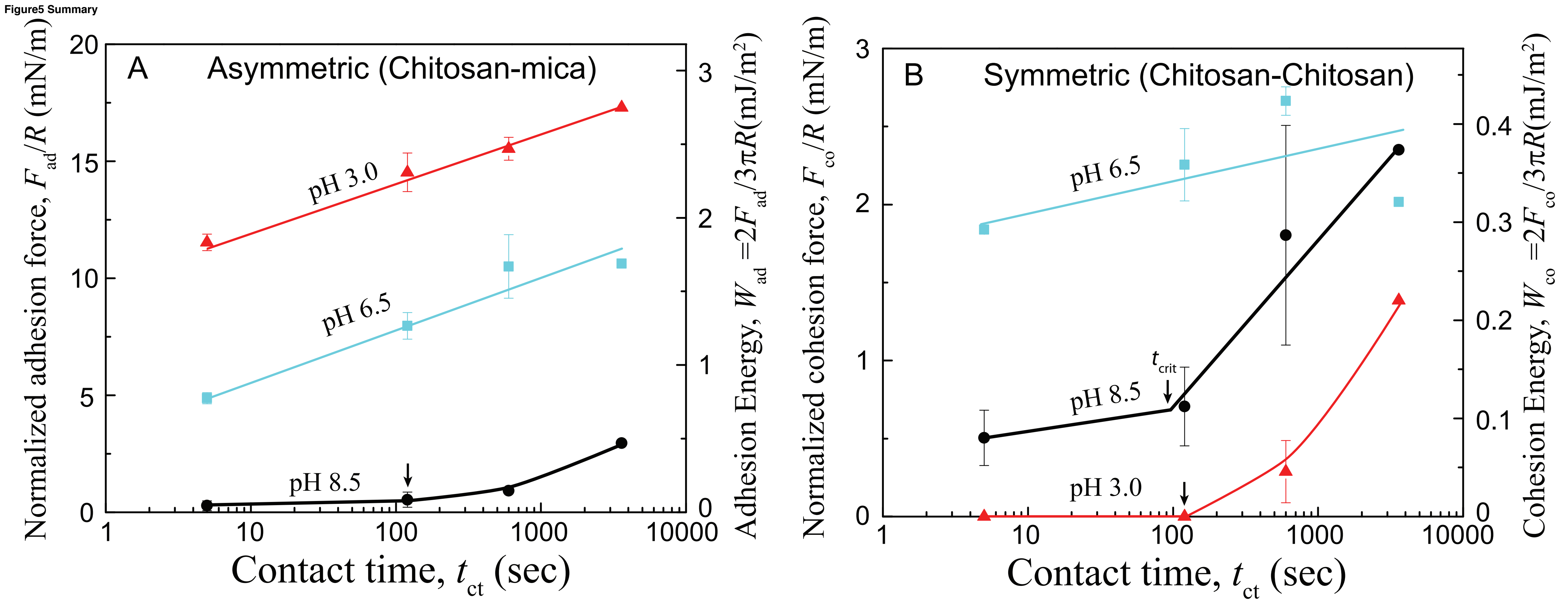

